\title{
Exosome-mediated protection of auditory hair cells from ototoxic insults
}

\author{
Ulrich Müller \\ Solomon H. Snyder Department of Neuroscience and Department of Biology, Johns Hopkins University School of Medicine, Baltimore, Maryland, USA.
}

\begin{abstract}
Hearing loss caused by the death of sensory hair cells of the inner ear is an unfortunate side effect for many patients treated with aminoglycoside antibiotics or platinum-containing chemotherapy agents. In animal models, induction of heat shock confers substantial otoprotection against aminoglycoside- and cisplatin-induced hair cell death. In this issue of the JCI, Breglio et al. demonstrate that inner ear tissue released exosomes carrying heat shock protein 70 (HSP70) in response to heat stress. HSP70 acted by a paracrine mechanism that engaged the Toll-like receptor 4 (TLR4) on hair cells to protect them from death. Exosomes and the HSP70/TLR4 pathway could thus provide treatment targets for the protection of hair cells from chemically induced death or from other insults, such as noise.
\end{abstract}

\section{Hair cells and ototoxicity}

Hearing loss is the most common form of sensory impairment in humans, affecting an estimated $5 \%$ to $10 \%$ of the population. Current treatments rely mostly on devices such as hearing aids and cochlear implants, and no FDA-approved drug for the condition is available. Genetic and environmental factors such as exposure to noise, infectious agents, and chemicals can cause hearing loss. However, some of the ototoxic chemicals also have substantial clinical value, which necessitates their use. These include antimicrobial aminoglycoside antibiotics, platinum-containing chemotherapy agents such as cisplatin, nonsteroidal antiinflammatory drugs like aspirin, loop diuretics like furosemide, and quinine, a major drug for malaria treatment. In the United States alone, approximately 500,000 patients annually experience damaging effects from treatment with ototoxic drugs (1). It is estimated that aminoglycosides alone cause hearing loss in $20 \%$ to $50 \%$ of patients treated for acute infections (2).
Animal model studies have demonstrated that treatment with aminoglycosides primarily damage sensory hair cells of the inner ear. These antibiotics are preferentially transported into the extracellular fluids of the inner ear and enter the hair cells through endocytosis and via mechanically gated ion channels $(2-5)$. In bacteria, aminoglycoside antibiotics bind to the 305 ribosomal subunit and disrupt protein synthesis. Mitochondrial ribosomes are similar to their bacterial counterparts, and it is thought that aminoglycosides cause hair cell death by inducing ribotoxic stress responses $(2,5)$. Other ototoxic drugs like cisplatin are also taken up by hair cells and cause their death (6). Given the relatively wide clinical use of ototoxic drugs, it is desirable to develop otoprotective strategies that maintain the therapeutic value of these drugs.

\section{Heat shock and otoprotection}

Cells in many tissues are protected from stress-induced apoptosis by the activation of heat shock proteins (HSPs). This protective effect is mediated at least in part

\section{Related Article: p. 2657}

Conflict of interest: Ulrich Müller is a co-founder of Decibel Therapeutics.

by the chaperone activity of HSPs and by direct inhibition of apoptotic signaling pathways (7). In the inner ear, activation of the heat shock response improves the survival of hair cells exposed to ototoxic drugs (8-10). To study the effect of the heat shock response on hair cells, the Cunningham laboratory adapted an organ culture system from the inner ear of mice (9). Hair cells in the inner ear are found in the cochlea, the end-organ for the perception of sound, as well as in the vestibule, the end-organ for the perception of gravity and acceleration (Figure 1). The hair cells of the cochlea and vestibule are morphologically and molecularly similar, and supporting cells that are critical for hair cell function surround both types of hair cells. Although explants from the cochlea are difficult to maintain in culture, culture conditions have been developed for vestibular explants.

Using such a culture system prepared from the utricle, a subcompartment of the vestibule, the Cunningham laboratory studied how innate stress response pathways, such as the heat shock response, protect hair cells from ototoxic drug insults (11). These foundational experiments demonstrated that utricular hair cells die rapidly following treatment with aminoglycosides but that pretreatment of the explants with heat has a protective effect, preventing considerable hair cell death (11).

In response to heat shock, the expression of HSP7O is induced in the organ explant, along with several other HSP proteins (9). However, heat shock failed to prevent hair cell death in organ explants generated from mice genetically engineered to lack expression of HSP70, indicating that among the HSP proteins, HSP7O is the most critical (11). Interestingly, HSP70 expression is induced in the supporting cells that surround the hair cells, but not in the hair cells themselves (11). This raises the question of how induction of HSP70 expression in supporting cells promotes hair cell survival. As one possibility, cytoplasmic HSP7O within supporting cells 


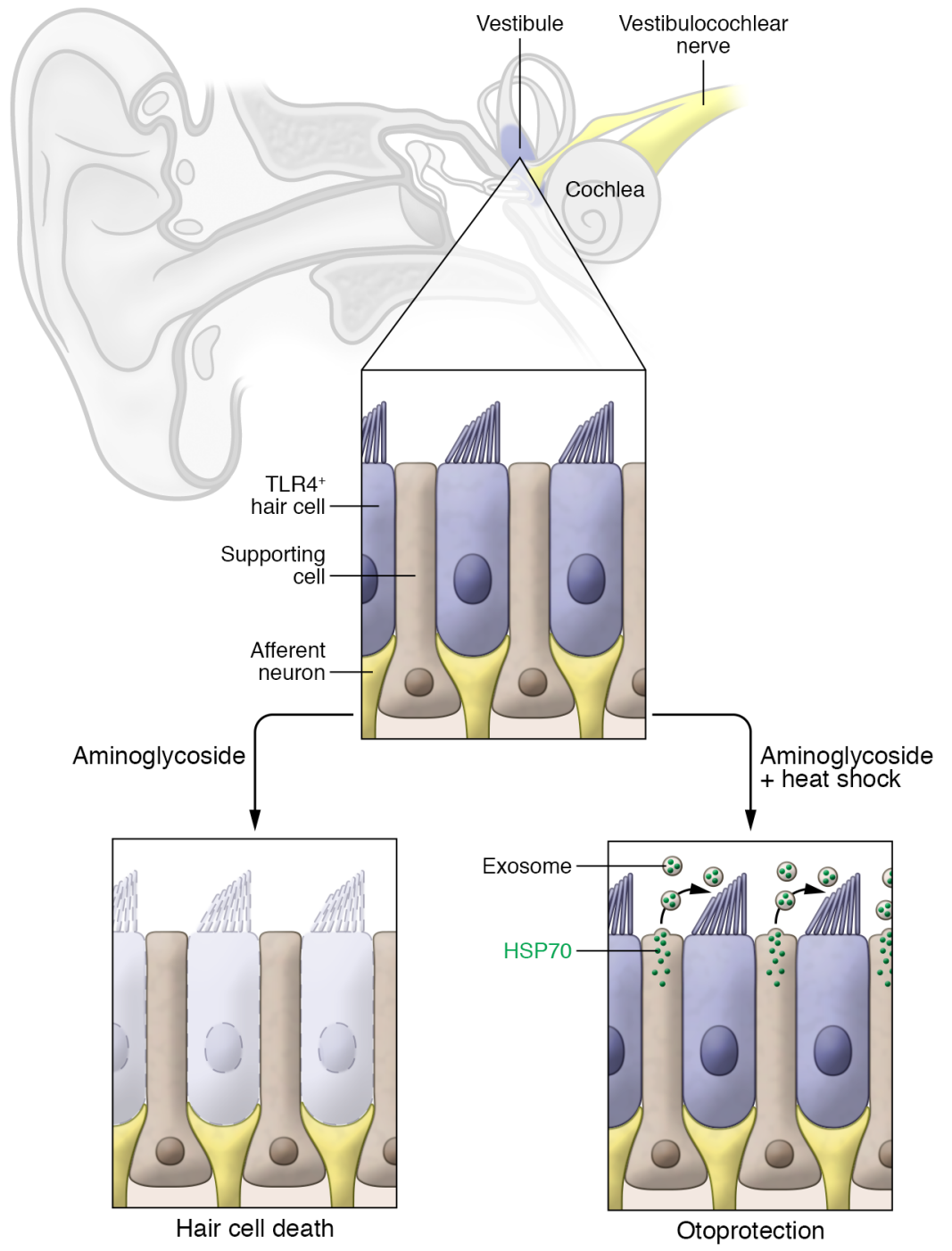

might activate a protective pathway. Alternatively, supporting cells might secrete HSP70 to affect hair cells directly. In support of the latter hypothesis, HSP70 was detected in the media of heat-shocked organ explants. Furthermore, heatshocked organ explants cocultured with non-heat-shocked explants protected hair cells in non-heat-shocked explants from death. When HSP7O was depleted from the media, the protective effect of heat shock was abolished (11). These experiments thus suggested that paracrine signaling from supporting cells to hair cells mediates the protective effect of HSP70 (Figure 1). However, the mechanisms by which HSP7O is released from supporting cells to protect hair cells were unknown. In this issue of the JCI, Breglio et al. have gone full circle and identified key mechanisms by which HSP70 affects hair cell survival (12).

\section{Paracrine exosome signaling protects hair cells}

It has long been established that cells can secrete proteins by the classical ER-Golgi pathway. However, HSP70 does not carry a signal sequence and is not shuttled through the ER-Golgi pathway. Cunningham and colleagues therefore set out to define the mechanism by which HSP70 is released from supporting cells. In the current issue of the JCI, Breglio et al. provide compelling evidence that supporting cells in the inner ear release HSP70 via exosomes (12). Exosomes are extracellular vesicles with a diameter of approximately 50 to $150 \mathrm{~nm}$ that are generated by the fusion of an intermediate endocytic compartment, the multivesicular body, with the plasma membrane (13). These vesicles are thought to be important for intracellular communication and
Figure 1. Exosomal HSP70-mediated protection of the mammalian inner ear. The mammalian inner ear contains the vestibule, which houses the sensory epithelia for the perception of gravity and head movements, and the cochlea, which houses the sensory epithelia for the perception of sound. Sensory epithelium from the utricle, a subcompartment of the vestibule, contains hair cells that are surrounded by supporting cells and innervated by afferent neurons. In utricle explant experiments, heat shock induced supporting cells to release exosomes containing HSP7O, which bound to TLR4-expressing hair cells and protected against aminoglycoside toxicity. can carry various payloads including proteins, lipids, and nucleic acids. Several laboratories have already provided evidence that cells release exosomes containing HSP70 in some tissues $(14,15)$. Colleagues from the Cunningham laboratory now demonstrate that heat shock induced the release of exosomes from inner ear tissue as well. These exosomes were released not only from supporting cells, but also from hair cells and other cells in the explants. Using tandem mass spectrometry, 291 protein families were identified in the exosomes, including known exosomal markers and one of the two inducible forms of HSP70 (12).

Breglio and colleagues then applied exosomes purified from heat-shocked explants to non-heat-shocked explants that had been treated with aminoglycosides. In the absence of the exosomes, hair cell 
death was massive, but exosome treatment protected hair cells. When exosome biogenesis was inhibited pharmacologically or when exosomes were treated with a function-blocking HSP70 antibody, the protective effect of heat shock was lost. Further, adding purified HSP70 protein to explants failed to provide an otoprotective effect, suggesting that presentation of HSP70 via exosomes is critical for otoprotection, or that exosomes contain additional molecules that cooperate with HSP70 in the protection of hair cells from death (12).

Colleagues from the Cunningham laboratory then went one step further. Using immunogold labeling, they localized HSP70 to exosomes and showed that HSP70 predominantly clustered around the exosomal membrane. HSP70 might thus localize appropriately in exosomes to interact with a receptor on the surface of hair cells. It was already known that HSP70 can bind to Toll-like receptor 4 (TLR4), a member of the TLR family that has critical roles in the recognition of pathogens and the activation of innate immunity (16). TLR4 had been implicated in protective roles mediated by HSP70, for example in heart and lung tissue $(17,18)$. Cunningham and colleagues generated a knockout mouse that specifically lacked TLR4 in hair cells. Consequently, HSP70 no longer protected hair cells from aminoglycoside-induced death (12).

The remarkable findings in the study by Breglio et al. outline a signaling pathway by which exosomes carrying HSP70 that are released from supporting cells bind to TLR4 on hair cells to protect them from aminoglycoside-induced death (12). The findings suggest that exosomes and the HSP70/TLR4 pathway could be valuable targets for the development of treatments that ameliorate the effects of chemically induced hair cell death. Although the experiments conducted by Breglio and colleagues focused on vestibular hair cells, it seems likely that similar mechanisms operate in the cochlea. In support of this exosome-HSP70/TLR4 model, celastrol, a pharmacological inducer of the heat shock response, prevents aminoglycoside-induced hair cell death and hearing loss in mice (19). Similar protective effects for auditory hair cells have been observed in transgenic mice overexpressing HSP7O (20).
The Breglio et al. study raises several interesting questions. First, what are the mechanisms that cause release of exosomes from supporting cells? A mechanistic understanding might provide entry points to trigger exosome release by pharmacological means. Notably, the Cunningham laboratory also demonstrated that purified HSP70 protein is not otoprotective (12). Do exosomes contain additional components besides HSP70 that are critical for otoprotection? The proteomics results presented in the manuscript might provide clues to other molecules that are involved. What is the role of TLR4 in otoprotection? Signaling pathways downstream of TLR4 have been explored in other tissues, but their role in the inner ear is unclear. Finally, could exosomes containing HSP70 be otoprotective against a variety of insults? Current evidence suggests that this might be the case. For example, exposure of mice to heat stress protects them from acoustic injury (21). Thus, exosomes might provide new venues for clinical intervention to protect hair cells in vivo from multiple stressors.

\section{Acknowledgments}

This work was supported by National Institute on Deafness and other Communication Disorders (NIDCD) grants DC005965, DC007704, and DC014713 (to UM), as well as a grant from the Rubenstein Fund for Hearing Research (to UM). UM is a Bloomberg Distinguished Professor.

Address correspondence to: Ulrich Müller, Room PCTB 1015, Solomon H. Snyder Department of Neuroscience, Johns Hopkins University School of Medicine, 725 North Wolfe Street, Baltimore, Maryland 21205, USA. Phone: 443.287.4762; Email: umuelle3@jhmi.edu.

1. Müller U, Barr-Gillespie PG. New treatment options for hearing loss. Nat Rev Drug Discovery. 2015;14(5):346-365.

2. Huth ME, Ricci AJ, Cheng AG. Mechanisms of aminoglycoside ototoxicity and targets of hair cell protection. Int JOtolaryngol. 2011;2011:937861.

3. Hailey DW, Esterberg R, Linbo TH, Rubel EW, Raible DW. Fluorescent aminoglycosides reveal intracellular trafficking routes in mechanosensory hair cells. J Clin Invest. 2017;127(2):472-486.
4. Marcotti W, van Netten SM, Kros CJ. The aminoglycoside antibiotic dihydrostreptomycin rapidly enters mouse outer hair cells through the mechano-electrical transducer channels. JPhysiol (Lond). 2005;567(Pt 2):505-521.

5. Warchol ME. Cellular mechanisms of aminoglycoside ototoxicity. Curr Opin Otolaryngol Head Neck Surg. 2010;18(5):454-458.

6. Langer T, am Zehnhoff-Dinnesen A, Radtke S, Meitert J, Zolk O. Understanding platinum-induced ototoxicity. Trends Pharmacol Sci. 2013;34(8):458-469.

7. Martindale JL, Holbrook NJ. Cellular response to oxidative stress: signaling for suicide and survival. J Cell Physiol. 2002;192(1):1-15.

8. Baker TG, et al. Heat shock protein-mediated protection against cisplatin-induced hair cell death. J Assoc Res Otolaryngol. 2015;16(1):67-80.

9. Cunningham LL, Brandon CS. Heat shock inhibits both aminoglycoside- and cisplatin-induced sensory hair cell death. JAssoc Res Otolaryngol. 2006;7(3):299-307.

10. Taleb M, Brandon CS, Lee FS, Lomax MI, Dillmann WH, Cunningham LL. Hsp70 inhibits aminoglycoside-induced hair cell death and is necessary for the protective effect of heat shock. JAssoc Res Otolaryngol. 2008;9(3):277-289.

11. May LA, et al. Inner ear supporting cells protect hair cells by secreting HSP70. J Clin Invest. 2013;123(8):3577-3587.

12. Breglio AM, et al. Exosomes mediate sensory hair cell protection in the inner ear. JClin Invest. 2020;130(5):2657-2672.

13. Colombo M, Raposo G, Théry C. Biogenesis, secretion, and intercellular interactions of exosomes and other extracellular vesicles. Annu Rev Cell Dev Biol. 2014;30:255-289.

14. De Maio A. Extracellular Hsp70: export and function. Curr Protein Pept Sci. 2014;15(3):225-231.

15. De Maio A, Vazquez D. Extracellular heat shock proteins: a new location, a new function. Shock. 2013;40(4):239-246.

16. Gay NJ, Gangloff M. Structure and function of Toll receptors and their ligands. Annu Rev Biochem. 2007;76:141-165.

17. Vicencio JM, et al. Plasma exosomes protect the myocardium from ischemia-reperfusion injury. JAm Coll Cardiol. 2015;65(15):1525-1536.

18. Zhang Y, Zhang X, Shan P, Hunt CR, Pandita TK, Lee PJ. A protective Hsp70-TLR4 pathway in lethal oxidant lung injury. JImmunol. 2013;191(3):1393-1403.

19. Francis SP, Kramarenko II, Brandon CS, Lee FS, Baker TG, Cunningham LL. Celastrol inhibits aminoglycoside-induced ototoxicity via heat shock protein 32. Cell Death Dis. 2011;2:e195.

20. Taleb M, Brandon CS, Lee FS, Harris KC, Dillmann WH, Cunningham LL. Hsp70 inhibits aminoglycoside-induced hearing loss and cochlear hair cell death. Cell Stress Chaperones. 2009;14(4):427-437.

21. Yoshida N, Kristiansen A, Liberman MC. Heat stress and protection from permanent acoustic injury in mice. J Neurosci. 1999;19(22):10116-10124. 